\title{
Cognitive skills improved by BrainWare SAFARI training program: Electroencephalographic study
}

\author{
Phakkharawat Sittiprapaporn \\ Assistant Professor and Head, Brain Science and Engineering Innovation Research Group, School of Anti-Aging and \\ Regenerative Medicine, Mae Fah Luang University, Bangkok. Thailand, and Department of Anti-Aging Science, School \\ of Anti-Aging and Regenerative Medicine, Mae Fah Luang University, Bangkok, Thailand
}

Background: Executive functions have been shown to be related to academic achievement and the ability to learn. Understanding the role of executive function may offer new learning opportunities for children. The 2018-2019 implementation of BrainWare SAFARI pilot study started with primary school students achieving an average improvement in cognitive skill development. Aims and Objective: This study was set to find spatial ability and cognitive skill abilities done by letting students who performed spatial ability tasks after BrainWare SAFARI training exercises and also investigated by using the effectiveness of commercially available lightweight EEG device. Materials and Methods: Participants were selected to participate in the 2018-2019 based on their average learning abilities. Results: All participants were able to complete the targeted number of sessions of BrainWare SAFARI. All participants showed significantly mean improvement of cognitive skills such as speed, memory, spatial ability, and problem solving, respectively. The cognitive performance increased statistically significant implying that all participants gained experienced significant improvements in multiple areas of cognitive processing. The attention and mediation levels as well as cognitive performance or cognitive skill improvement were changed with statistically significant at 0.05 level. The findings also showed that all brainwaves were increased with statistically significant while performing cognitive skills ability tasks. Conclusion: It is no doubt that all participants were able to complete all sessions of BrainWare SAFARI program. Practicing cognitive skills improvement games as BrainWare SAFARI could significantly increase alpha, theta and beta frequency bands. BrainWare SAFARI cognitive skills training could significantly improve cognitive performance in Thai children indexed by the electroencephalographic activities.

Key words: Brain; BrainWare SAFARI; Cognitive development; Learning; Student

\section{Access this article online}

Website:

http://nepjol.info/index.php/AJMS DOI: 10.3126/ajms.v11i1.26526 E-ISSN: 2091-0576

P-ISSN: $2467-9100$

\section{INTRODUCTION}

Brain is an organ that is easily affected by the physical changes. Abnormalities will occur to the brain in terms of memory, eyesight, intellect, and balance. Because of these damages, people should know how to protect themselves from the poisonous substances. ${ }^{1-3}$ Cognitive abilities including domain knowledge, crystallized intelligence, memory, and psychomotor speed were used in investigating the success in computer-based cognitive training programs. ${ }^{4}$ Previous studies used computer-based cognitive skill training in improving the cognitive abilities of students diagnosed with specific learning disabilities. ${ }^{5-10}$ These previous studies showed that computer-based training can be effective in improving executive functions and working memory. ${ }^{5,7,11}$ Moreover, some previous studies used computer-based cognitive training programs to examined the improvement of working memory of adults and students. ${ }^{5,8,10}$ For instance, in Westerberg et al.'s study, it was found that computer-based cognitive raining

Address for Correspondence:

Dr. Phakkharawat Sittiprapaporn, Assistant Professor and Head, Brain Science and Engineering Innovation Research Group, School of AntiAging and Regenerative Medicine, Mae Fah Luang University, Bangkok, Thailand. Tel. No.: +662 6644361. E-mail: wichian.sit@mfu.ac.th (c) Copyright AJMS 
program improved the working memory of adult patients who had stroke within the past year. ${ }^{10}$ Using students as the sample, Aleven and Koedinger ${ }^{5}$ and Thorell et al. ${ }^{9}$ found similar effectiveness of computer-based cognitive training programs in improving working memory. Additionally, Olesen and his colleagues found similar results regarding the effectiveness of computer-based cognitive raining programs in healthy adults. ${ }^{8}$

BrainWare SAFARI is a software program which is designed to comprehensively develop the cognitive skills in children. These cognitive skills are most important for learning. BrainWare SAFARI delivers cognitive skills in a fun and entertaining video-game format. Several previous published researches showed that students who used BrainWare SAFARI experienced significant improvements in their cognitive skills. In addition, a growing body of research shows the connection between the development of cognitive skills and academic progress. Academic progress typically lags cognitive growth and the rate of gain in academic achievement is highly individual. Moreover, some students experienced an immediate lift in academic scores, while others might have significant catching-up to do because they need to learn or re-learn material they missed. Therefore, this study was set to find spatial ability and cognitive skill abilities done by letting participants who performed spatial ability tasks after BrainWare SAFARI training exercises and also investigated by using the effectiveness of commercially available lightweight Electroencephalographic (EEG) device.

\section{MATERIALS AND METHODS}

\section{Participants}

The experiment was done with twenty students, aged between 6-12 years old, who were in good health, no congenital illness, no record of brain surgery, not taking medicines or drugs that affect to nervous system. Participants were excluded if they had the following criteria: history of medical and neurologic diseases; psychiatric disorders; head trauma; assumption of Central Nervous System active drugs in the two weeks prior to study entry; and presence of EEG abnormalities at the baseline recording, respectively. All participants' parents provided written consent to participate in the study after receiving information about the aims of the study.

\section{Tools and equipment}

Recording personal information, namely, age, gender, nationality, and history of illness were recorded. The electroencephalographic recording was applied in this study. The neuroheadset was used to display the output, analyze and record electroencephalographic activities. The principles of analytical check were based on the 10-20 system or International 10-20 system by displaying the electroencephalographic output. Electroencephalographic frequency analysis was performed by means of a Fast Fourier Transform (FFT) algorithm, with a 2 -second interval on the electroencephalographic signal. The following frequency bands were considered: delta wave $(0.1-3.0 \mathrm{~Hz})$; theta wave $(4.0-7.0 \mathrm{~Hz})$; alpha wave $(8.0-12.0 \mathrm{~Hz})$; beta wave $(13.0-30.0 \mathrm{~Hz})$; and gamma wave $(30.0-60.0 \mathrm{~Hz})$, respectively.

In this study, the effectiveness of commercially available lightweight electroencephalographic devices, NeuroSky's Mindwave Mobile, was applied. Via the application of a single electrode and signal-processing unit in a headband arrangement, the NeuroSky's Mindwave Mobile provided two 100-state outputs operating at $1 \mathrm{~Hz}$. Although the NeuroSky's Mindwave Mobile provided a much coarser picture of brain activity than multi-electrode electroencephalographic device or other technologies, the principle advantage of the NeuroSky's Mindwave Mobile was its unobtrusive nature, which minimizes the aforementioned difficulties in conducting accurate user studies.

\section{Spatial ability test}

Spatial ability test used in this study is known as the test to measure capacity to understand, reason and remember the spatial relations among objects or space. ${ }^{12}$ Spatial or visuo-spatial perception, spatial visualization, mental folding and mental rotation are common types of spatial abilities. ${ }^{12}$ However, only spatial visualization is known to be characterized as complicated multi-step manipulations of spatially presented information. In the same way, spatial working memory is known as the ability to temporarily store a certain amount of visual-spatial memories under attentional control in order to complete a task. ${ }^{13-14} \mathrm{Each}$ of four main spatial abilities theoretically have unique properties and importance to many types of tasks whether in certain jobs or everyday life ${ }^{12}$ (Figure 1).

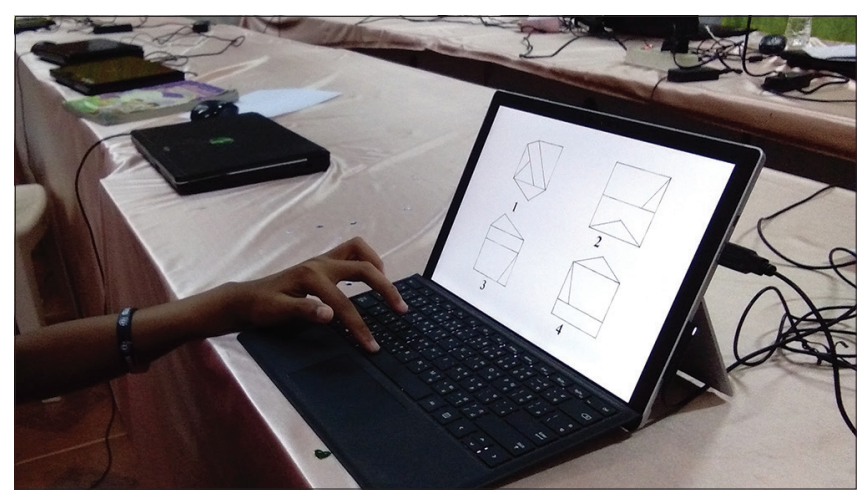

Figure 1: One of participants performed the spatial ability task after training with BrainWare SAFARI Program 
Cognitive training program

BrainWare SAFARI was a cognitive learning program which was designed to comprehensively develop the cognitive skills. As these cognitive skills are important for learning, the BrainWare SAFARI program delivered in a fun and entertaining video-game format. BrainWare SAFARI consisted of 20 exercises designed to develop various cognitive skills. In 18 of the exercises, each with seven levels, levels were progressively more difficult. Each level must be passed before the student could progress to the next level. The two logic and reasoning exercises consisted of 21 levels each and were of approximately the same level of difficulty, allowing the students to see the right answer and move on after two incorrect answers (Figure 2). As BrainWare SAFARI required an internet connection, it was designed as a rich-client application to enhance the user's experience. In addition, the log-in and progress through the program were controlled by LEC's server. This system enabled the player to always begin in the exercises where he or she left off, regardless of whether he or she is on the same computer. BrainWare SAFARI program is developed by BrainWare Learning Company and more details of BrainWare SAFARI can be seen at www.mybrainware.com.

\section{Electroencephalographic recording}

Measuring electroencephalographic activities while performing spatial ability tests run by SuperLab Pro. Version 2.0 (Cedrus Corporation, San Pedro, USA), stimuli presentation, compared to baseline. SuperLab Pro was used to develop spatial ability tests for electroencephalographic measurement. A single-channel electroencephalogram was designed to collect brain activity data from the participants' brain. Simplicity and efficiency were the main reasons for designing a single-channel electroencephalographic monitoring system. Electroencephalographic data was analyzed offline and the following frequency bands were computed: delta wave ranging from 0.1 to $3.0 \mathrm{~Hz}$, theta wave $(4.0$ to $7.0 \mathrm{~Hz})$, alpha wave $(8.0$ to $13.0 \mathrm{~Hz})$, beta wave $(14.0$ to $30.0 \mathrm{~Hz})$, and gamma wave $(31.0$ to $47.0 \mathrm{~Hz})$, respectively.

\section{Statistical analysis}

The quantitative data was analyzed by mean of average and standard deviation. Using inferential statistical analysis to compare each type of brainwaves by compared $t$-test. Every test was set to the statistic significant at $p<0.05$.

\section{RESULTS}

In this study, the attention and mediation levels were measured both before and after BrainWare SAFARI training program. The result showed that the attention level changed with statistically significant at 0.05 level $(p$-value $=0.05)$. The average before training was 73.29
$( \pm .78)$ score and the average after training was 84.41 $( \pm 0.93)$ score. For meditation level, there was changed with statistically significant at 0.05 level $(p$-value $=0.05)$. The average before training was $54.38( \pm 0.73)$ score and the average after training was $76.33( \pm 0.15)$ score. In average of both attention and meditation levels, the cognitive performance or cognitive skill improvement was calculated. There was changed with statistically significant at 0.05 level $(p$-value $=0.05)$. The average before training was $63.84( \pm 0.37)$ score and the average after training was $80.37( \pm 0.42)$ score (Table 1 and Figure 3$)$.

Based on Table 2, it was found that all brainwaves gradually increased while theta, alpha, and beta brainwaves were changed with statistically significant at the 0.05 level. Even though both delta and gamma brainwaves were increased, but they were changed with no statistically significant while performing spatial ability tasks (Figure 4).

According to Table 2 and Figure 4, it was found that delta brainwave increased, but with no statistically significant

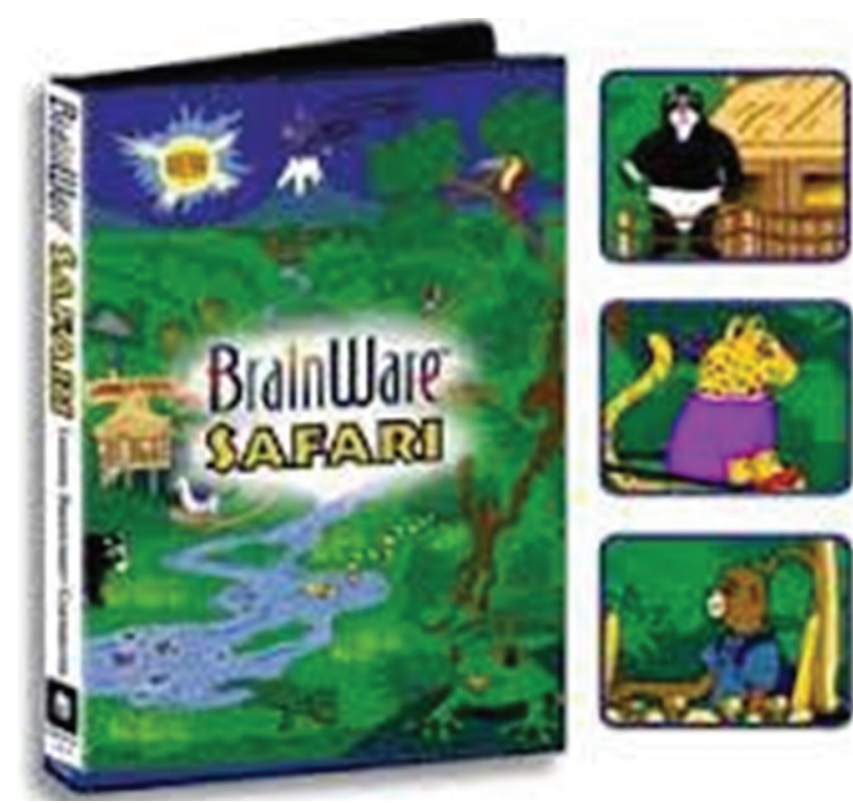

Figure 2: BrainWare SAFARI Program by BrainWare Learning Company

\begin{tabular}{|c|c|c|c|c|c|}
\hline \multirow{2}{*}{$\begin{array}{l}\text { Cognitive } \\
\text { abilities }\end{array}$} & \multicolumn{2}{|c|}{ Before training } & \multicolumn{2}{|c|}{ After training } & \multirow[t]{2}{*}{$p$-value } \\
\hline & Mean & SD & Mean & SD & \\
\hline Attention & 73.29 & 0.78 & 84.41 & 0.93 & $0.05^{*}$ \\
\hline Meditation & 54.38 & 0.73 & 76.33 & 0.15 & $0.05^{*}$ \\
\hline $\begin{array}{l}\text { Cognitive } \\
\text { Performance }\end{array}$ & 63.84 & 0.37 & 80.37 & 0.42 & $0.05^{*}$ \\
\hline
\end{tabular}




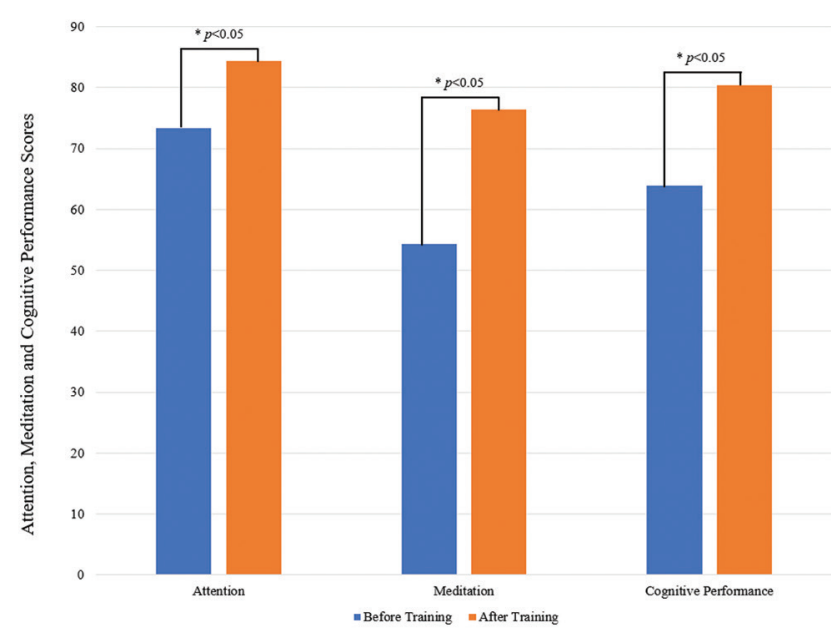

Figure 3: Attention and mediation levels as well as cognitive performance were measured both before and after BrainWare SAFARI training program

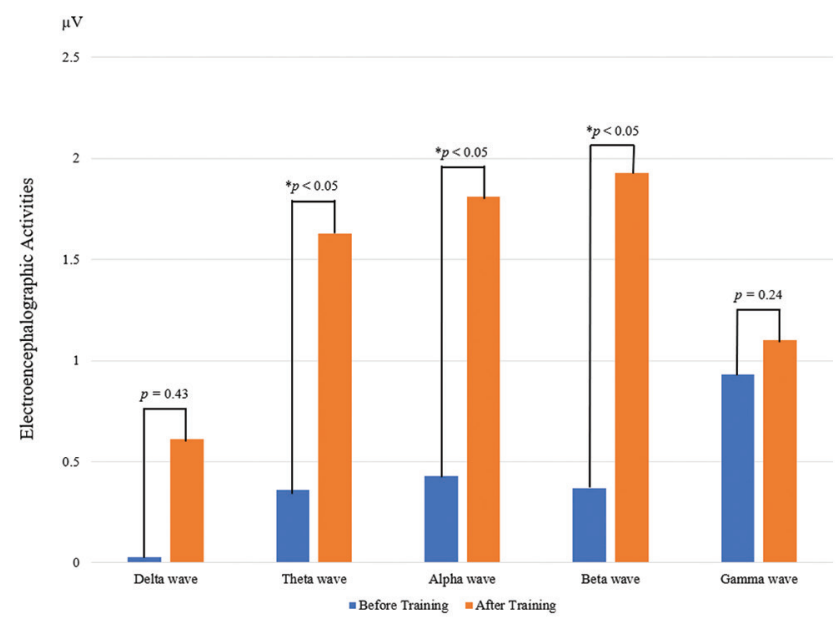

Figure 4: Electroencephalographic activities while peforming spatial ability tasks both before and after BrainWare SAFARI training program

\begin{tabular}{|c|c|c|c|c|c|}
\hline \multirow{2}{*}{$\begin{array}{l}\text { EEG } \\
\text { activities }\end{array}$} & \multicolumn{2}{|c|}{ Before training } & \multicolumn{2}{|c|}{ After training } & \multirow[t]{2}{*}{$p$-value } \\
\hline & Mean & SD & Mean & SD & \\
\hline $\begin{array}{l}\text { Delta } \\
\text { brainwave }\end{array}$ & 0.03 & 0.15 & 0.61 & 0.73 & 0.43 \\
\hline $\begin{array}{l}\text { Theta } \\
\text { brainwave }\end{array}$ & 0.36 & 0.07 & 1.63 & 0.04 & $0.01^{*}$ \\
\hline $\begin{array}{l}\text { Alpha } \\
\text { brainwave }\end{array}$ & 0.43 & 0.18 & 1.81 & 0.12 & $0.01^{*}$ \\
\hline $\begin{array}{l}\text { Beta } \\
\text { brainwave }\end{array}$ & 0.37 & 0.29 & 1.93 & 0.07 & $0.01^{*}$ \\
\hline $\begin{array}{l}\text { Gamma } \\
\text { brainwave }\end{array}$ & 0.93 & 0.25 & 1.10 & 0.19 & 0.24 \\
\hline
\end{tabular}

while performing spatial ability tasks after BrainWare SAFARI training compared to baseline (before training:
$0.03 \pm 0.15 \mu \mathrm{V}$; after training: $0.61 \pm 0.73 \mu \mathrm{V} ; \mathrm{t}(19)=1.27$; $p=0.43)$. Similar to delta brainwave, theta brainwave increased with statistical significant at the 0.01 level while performing spatial ability tasks after BrainWare SAFARI training compared to baseline (before training: $0.36 \pm 0.07 \mu \mathrm{V}$; after training: $1.63 \pm 0.04 \mu \mathrm{V} ; \mathrm{t}(19)=0.87$; $p=0.01)$.

In the same way, it was found that alpha brainwave was found to be increased with statistically significant at the 0.01 level while performing spatial ability tasks after BrainWare SAFARI training compared to baseline (before training: $0.43 \pm 0.18 \mu \mathrm{V}$; after training: $1.81 \pm 0.12 \mu \mathrm{V} ; \mathrm{t}(19)=0.61$; $p=0.01)$. Moreover, beta brainwave also increased with statistically significant at the 0.01 level while performing spatial ability tasks after BrainWare SAFARI training compared to baseline (before training: $0.37 \pm 0.29 \mu \mathrm{V}$; after training: $1.93 \pm 0.07 \mu \mathrm{V} ; \mathrm{t}(19)=0.37 ; p=0.01)$. Finally, it was found that even though gamma brainwave increased, but it was not increased statistically significant while performing spatial ability tasks after BrainWare SAFARI training compared to baseline (before training: 0.93 $\pm 0.25 \mu \mathrm{V}$; after training: $1.10 \pm 0.19 \mu \mathrm{V} ; \mathrm{t}(19)=1.77 ; p=0.24)$.

\section{DISCUSSION}

In general observation, all participants were able to complete the targeted number of sessions of BrainWare SAFARI. All participants showed significantly mean improvement of cognitive skills such as speed, memory, spatial ability, and problem solving, respectively. Additionally, the attention and mediation levels as well as a cognitive performance or cognitive skills improvement measured by lightweight Electroencephalographic device were also monitored either before or after training while performing spatial ability tasks. In this study, the cognitive performance increased statistically significant implying that all participants gained experienced significant improvements in multiple areas of cognitive processing. The findings also showed that all brainwaves were increased with statistically significant while performing spatial ability tasks.

According to several previous studies, spatial abilities were called as the mental capacities composing of construction, transformation and interpretation of mental images. These abilities reflect the use of mental images in order to manipulate spatial representations. ${ }^{15}$ A number of factors have been proposed including visualization, orientation and spatial relations, respectively. In addition, the spatial relation ability corresponds to the capacity to rapidly and accurately rotate a mental image. ${ }^{15}$ Several studies have been tried to determine the different factors composing the spatial abilities. ${ }^{15}$ The finding of present study was consistent 
with those findings in several previous studies including in healthy adults ${ }^{12,16-17}$. Moreover, electroencephalographic activities while performing spatial ability tasks showed significant increase in delta, theta and alpha brainwaves whereby the findings were also consistent with previous studies showing that the sensorimotor rhythm related to attention. ${ }^{13-14,18}$

Electroencephalography (EEG) is traditionally used to measure the brainwaves. Each type of brainwave is associated with one's state of consciousness and different mood state. ${ }^{19-22}$ From all types of brainwave, beta brainwave is seen in normal activities as well as stressful conditions or difficulties in mental concentration. On the other hand, alpha brainwave is seen in wakefulness, relaxed, effortless and alertness condition. Therefore, persons with high alpha brainwave and low in beta brainwave could indicate status of relaxation, arousal, less stress and better concentration. $^{23-26}$

A brain computer interface (BCI) is a hardware and software communication system that enables its user to interact with surroundings without the involvement of peripheral nerves and muscles, i.e., by using control signals generated from electroencephalographic activities. ${ }^{27}$ In the present study, all participants have shown significantly mean improvement in electroencephalographic activities and some areas of cognitive functions for instance memory, attention, and problem solving etc. Electroencephalographic activities after practicing spatial ability and cognitive skills training via BrainWare SAFARI in the present study showed significant increase in alpha, theta and beta frequency bands. Even though this study was inconsistent with the previous studies i.e., Egner and Gruzelier ${ }^{28}$ and Rossiter and LaVaque ${ }^{29}$ found that SMR (Sensorimotor Rhythm) or lower beta brainwave related to attention, we found a relation between theta brainwave and memory which was consistent with Egner and Gruzelier and Rossiter and LaVAque studies. ${ }^{28-29}$ Furthermore, the finding in the present study stayed in the line of previous studies on brain training program which has been shown to be efficient in many different areas especially in the mathematics and engineering education. ${ }^{18,30}$ Consequently, practicing the spatial ability and cognitive processing skills in BrainWare SAFARI in the present study could significantly increase all type of brainwaves. Thus, BrainWare SAFARI training program could help significantly improve cognitive performance indexed by the electroencephalographic activities.

\section{CONCLUSION}

It is no doubt that all participants involved in this study were able to complete all sessions of BrainWare SAFARI program. Those completed a sufficient number of sessions in BrainWare SAFARI showed significant improvements in all multiple areas of cognitive processing in BrainWare SAFARI. Practicing cognitive skills improvement games as BrainWare SAFARI could significantly increase alpha, theta and beta frequency bands. BrainWare SAFARI cognitive skills training could significantly improve cognitive performance in Thai children indexed by the electroencephalographic activities.

\section{ACKNOWLEDGEMENT}

This research was supported by research funding from Asia Research Center, Chulalongkorn University, Bangkok, Thailand, and The Korea Foundation for Advanced Studies (KFAS), Korea. Authors gratefully acknowledge Mae Fah Luang University grant (Electroencephalogram Laboratory 2019), Mae Fah Luang University grant (MFU-grant no. 2201A601814), and Brain Science and Engineering Innovation Research Group, Mae Fah Luang University grant (MFU-grant no. 611U109005) for the support and necessary provision. We thank all of the subjects involved in this research as main data provider of this studies.

\section{REFERENCES}

1. Bailey SP, Hall EE, Folger A and Miller PC. Changes in EEG during graded exercise on a recumbent cycle ergometer. $\mathrm{J}$. Sports Sci Med 2008; 7: 505-511.

2. Gutmann B, Mierau A, Hulsdunker T, Hildebrand C, Przyklenk A, Hollmann W, et al. Effects of Physical Exercise on Individual Resting State EEG Alpha Peak Frequency. Neural Plast 2015; 717312.

3. Moraes $H$, Deslandes $A$, Silveira $H$, Ribeiro $P$, Cagy $M$, Piedade R, et al. The effect of acute effort on EEG in healthy young and elderly subjects. Eur J Appl Physiol 2011; 111(1): 67-75.

4. Ownby R, Czaja SJ, Loewenstein D and Rubert M. Cognitive abilities that predict success in a computer-based training program. Gerontologist 2008; 48(2): 170-180.

5. Aleven VAWMM and Koedinger KR. An effective metacognitive strategy: Learning by doing and explaining with a computerbased cognitive tutor. Cognitive Science 2002; 26: 147-179.

6. Hung J, Randolph-Seng B, Monsicha K and Crooks SM. Computer-based instruction and cognitive load. [Online] 2018 [Citation April 17, 2019] from http://scholarworks.boisestate.edu/ cgi/viewcontent.cgi?article $=1004 \&$ context=edtech_facpubs

7. Mezzacappa E and Buckner JC. Working memory training for children with attention problems or hyperactivity: A school-based pilot study. School Mental Health: A Multidisciplinary Research and Practice Journal 2010; 2(4): 202-208.

8. Olesen PJ, Westerberg $\mathrm{H}$ and Klingberg $\mathrm{T}$. Increased prefrontal and parietal activity after training of working memory. Nature Neuroscience 2002; 11: 1-5.

9. Thorell LB, Lindqvist S, Bergman S, Bohlin G and Klinberg T. Training and transfer effects of executive functions in preschool children. Developmental Science. 2008; 11(6): 969-976.

10. Westberg $H$, Jacobaeus $H$, Hirvikoski $T$, Clevberger $P$, 
Ostensson ML and Bartfai A. Computerized working memory training after stroke-A pilot study. Brain Injury. 2007; 21(1): 21-29.

11. Floyd RG, Bergeron R, Hamilton G and Parra GR. How do executive functions fit with the Cattell-Horn-Carrroll model? Some evidence from a joint factor analysis of the Delis-Kaplan executive function system and the Woodcock-Johnson III tests of cognitive abilities. Psychology in the Schools 2010; 47(7): 721-738.

12. Kessler S, Hosseini $H$, Heckler $C$, Janelsins M, Palesh $O$, Mustian K, et al. Cognitive Training for Improving Executive Function in Chemotherapy-Treated Breast Cancer Survivors. Clin. Breast Cancer 2013; 13(4): 299-306.

13. Egner $\mathrm{T}$ and Gruzelier $\mathrm{JH}$. Learned self-regulation of EEG frequency components affects attention and event-related brain potentials in humans. Neuro Report 2001; 12: 4155-4159.

14. Rossiter R and LaVaque TJ. A comparison of EEG biofeedback and psychostimulants in treating attention deficit hyperactivity disorders. Neurother 1995; 4: 48-59.

15. Poltrock SE and Brown P. Individual differences in visual imagery and spatial ability. Intelligence 1984; 8: 93-138.

16. Joshep HL, David D, Kumal S, Gregory K and Michael S. Enhancing visual attention and working memory with a webbased cognitive training program. Mensa Res 2011; 42(2): 13-20.

17. Finn $\mathrm{M}$ and McDonald J. Improvement in visual sustained attention in older adults with mild cognitive impairment. Australian Ageing 2010; 29(Supplement 2): 15.

18. Vandenberg SG and Kuse AR. Mental rotations, a group test of three-dimensional spatial visualization. Percept. Motor Skills 1978; 47: 599-604.

19. Allen JJB, Coan JA and Nazarian M. Issues and assumptions on the road from raw signals to metrics of frontal EEG asymmetry in emotion. Biol. Psychol.2004; 67: 183-218.

20. Crabbe JB and Dishman RK. Brain electrocortical activity during and after exercise: A quantitive synthesis. Psychophysiol 2004; 41: 563-574.

21. Davis JM and Bailey SP. Possible mechanisms of central nervous system fatigue during exercise. Med. Sci. Sport and Exer 1997; 29(1): 45-57.

22. Kakizaki T. Effects of bicycle exercise on occipital EEG amplitude in male students', Industrial Health 1988; 26: 191-195.

23. Kamijo K, Nishihira Y, Hatta A, Kaneda T, Kida T, Higashiura T, et al. Changes in arousal level by differential exercise intensity. Clin Neurophysiol 2004; 115: 2693-2698.

24. Kraaier V, Van Huffelen $A C$ and Wieneke GH. Changes in quantitative EEG and blood flow velocity due to standardized hyperventilation; a model of transient ischaemia in young human subjects. Electroencephal Clin Neurophysiol 1988; 70: 377-387.

25. Kraaier V, Van Huffelen AC, Wieneke GH, Van der Worp HB and Bär PR. Quantitative EEG changes due to cerebral vasoconstriction. Indomethacin versus hyperventilationinduced reduction in cerebral blood flow in normal subjects. Electroencephal Clin Neurophysiol 1992; 82: 208-212.

26. Kubitz KA and Pothakos K. Does aerobic exercise decrease brain activation? J. Sport and Exer Psychol 1997; 19: 291-301.

27. Bailey SP, Hall EE, Folger A and Miller PC. Changes in EEG during graded exercise on a recumbent cycle ergometer. J Sports Sci Med 2008; 7: 505-511.

28. Egner $\mathrm{T}$ and Gruzelier $\mathrm{JH}$. Learned self-regulation of EEG frequency components affects attention and event-related brain potentials in humans. Neuro Report 2001; 12: 4155-4159.

29. Rossiter $R$ and LaVaque TJ. A comparison of EEG biofeedback and psychostimulants in treating attention deficit hyperactivity disorders. J Neurother 1995; 4: 48-59.

30. Bailey SP, Hall EE, Folger A and Miller PC. Changes in EEG during graded exercise on a recumbent cycle ergometer. J Sports Sci Med 2008; 7: 505-511.

\footnotetext{
Authors Contribution:

PS- Concept and design of the study, statistically analyzed and interpreted, manuscript preparation, critical revision of the manuscript.

Work attributed to:

Brain Science and Engineering Innovation Research Group, School of Anti-Aging and Regenerative Medicine, Mae Fah Luang University, Thailand.

Orcid ID:

Dr. Phakkharawat Sittiprapaporn - (1) https://orcid.org/0000-0002-4103-9396

Source of Support: Asia Research Center, Chulalongkorn University, Bangkok, Thailand, and The Korea Foundation for Advanced Studies (KFAS), Korea; Mae Fah Luang University grant (Electroencephalogram Laboratory 2019), Mae Fah Luang University grant (MFU-grant no. 2201A601814), and Brain Science and Engineering Innovation Research Group, Mae Fah Luang University grant (2019) (MFU-grant no. 611U109005), and 2020 Thailand, Conflict of Interest: None.
} 